\title{
INFINITESIMALLY STABLE ENDOMORPHISMS
}

\author{
HIROSHI IKEDA
}

\begin{abstract}
It is well known that infinitesimal stability of diffeomorphisms is an open property. However, infinitesimal stability of endomorphisms is not an open property. So we consider the interior of the set of all infinitesimally stable endomorphisms. We prove that if $f$ belongs to the interior of the set of all infinitesimally stable endomorphisms, then $f$ is $\Omega$-stable. This means a generalization of Smale's $\Omega$-stability theorem for diffeomorphisms. Moreover, it is proved that for Anosov endomorphisms structural stability is equivalent to lying in the interior of the set of infinitesimally stable endomorphisms.
\end{abstract}

\section{INTRODUCTION}

In the theory of dynamical systems structural stability is one of important concepts. Moreover infinitesimal stability is closely related to structural stability. For example, in the diffeomorphisms theory the following result has been known.

Theorem A $[4,7,16]$. Let $f$ be a $C^{1}$ diffeomorphism of a closed smooth manifold $M$. Then the following are equivalent:

(a) $f$ is $C^{1}$ structurally stable;

(b) $f$ satisfies Axiom $\mathrm{A}$ and the strong transversality condition;

(c) $f$ is infinitesimally stable.

Now we shall attempt to give an outline of the development of infinitesimal stability without giving precise definitions. In the case of diffeomorphisms, Robbin [14], inspired by Moser [9], introduced the concept of infinitesimal stability to prove the structural stability theorem. At first, infinitesimal stability was a medium to prove structural stability; i.e., (b) $\Rightarrow(\mathrm{c}) \Rightarrow(\mathrm{a})$. Moreover Mañé [4] generalized this concept and proved that (b) is equivalent to (c). In this paper the concept of infinitesimal stability of diffeomorphisms means Mañés generalized version. From the result of Mañé [7], we now know that for $C^{1}$ diffeomorphisms infinitesimal stability is equivalent to structural stability. The concept of infinitesimal stability of endomorphisms virtually appeared in [5]. Using the concept we obtained properties similar to those of infinitesimally stable diffeomorphisms [2, 3]. In [3] the following question was stated:

Question. Is infinitesimal stability of endomorphisms an open property?

Received by the editors October 12, 1992 and, in revised form, September 28, 1993.

1991 Mathematics Subject Classification. Primary 58F10; Secondary 58F15.

Key words and phrases. Infinitesimal stability, Axiom A, prehyperbolic set, $\Omega$-stability. 
For this question Odani obtained a negative answer [11]. It is evident that infinitesimal stability of diffeomorphisms is an open property. So we consider the interior of the set of all infinitesimally stable endomorphisms. It appears that lying in the interior of the set of all infinitesimally stable endomorphisms is appropriate to a generalization of infinitesimal stability of diffeomorphisms. Hence one would like to conjecture that lying in the interior of the set of all infinitesimally stable endomorphisms is equivalent to structural stability. A motivation of this conjecture is that for $C^{1}$ diffeomorphisms infinitesimal stability is equivalent to structural stability. Another is that research for seeking above equivalence may be a key of finding.appropriate necessary and sufficient conditions for endomorphisms to be structurally stable. In this paper we give the following results which are a start to prove the above conjecture.

Theorem I. Let $f$ be a $C^{r}$ endomorphism of a closed smooth manifold $M$, $r \geq 1$. If $f$ belongs to the interior of the set of all $C^{r}$ infinitesimally stable endomorphisms of $M$, then $f$ is $\Omega$-stable.

Corollary I. Let $f$ be a $C^{r}$ endomorphism of a closed smooth manifold $M$, $r \geq 1$. If $f$ has a $C^{r}$ neighborhood $\mathscr{U}$ of $f$ such that every $g \in \mathscr{U}$ satisfies weak Axiom A, then $f$ is $\Omega$-stable.

Theorem II. Let $f$ be a $C^{r}$ Anosov endomorphism of a closed smooth manifold $M, r \geq 1$. Then the following are equivalent:

(a) $f$ is $C^{r}$ structurally stable;

(b) $f$ is either an Anosov diffeomorphism or an expanding map;

(c) $f$ belongs to the interior of the set of all infinitesimally stable endomorphisms of $M$.

The above corollary gives sufficient condition for $\Omega$-stability. Przytycki [13] provided sufficient conditions for an endomorphism $f$ to be $\Omega$-stable. His conditions require that the nonwandering set of $f$ contains no singularities. However, there exist examples of $\Omega$-stable endomorphisms with singularities in the nonwandering set [8]. Our sufficient condition permits the existence of singularities in the nonwandering set. Moreover this corollary is a natural generalization of Smale's $\Omega$-stability theorem [19].

The contents of this paper are as follows: In $\S 2$ we establish some background and give the definition of infinitesimal stability of endomorphisms. In $\S 3$ we prove Theorem I and relate this result with Smale's $\Omega$-stability theorem. In $\S 4$ we give the definition of Anosov endomorphisms and prove Theorem II. Section 5 contains some concluding remarks.

\section{Preliminaries}

Let $M$ be a compact connected smooth manifold without boundary and let $\operatorname{End}^{r}(M), r \geq 1$, be the space of $C^{r}$ endomorphisms of $M$ endowed with the $C^{r}$ topology. For $f \in \operatorname{End}^{r}(M)$, the nonwandering set of $f$ is denoted by $\Omega(f)$ and is defined to be the set of all $x \in M$ such that for every neighborhood $U$ of $x$ there exists an integer $n>0$ with $f^{n}(U) \cap U \neq \varnothing$. The set of periodic points of $f$ is denoted by $\operatorname{Per}(f)$. We denote by $S(f)$ the set of singularities of $f$, i.e. those points $x$ of $M$ where $T f \mid T_{x} M$ is not injective. We say that an endomorphism $f$ is $C^{r}$ structurally (resp. $\Omega$-) stable if there exists a 
neighborhood $\mathscr{U}$ of $f$ in $\operatorname{End}^{r}(M)$ such that for every $g$ in $\mathscr{U}$ there exists a homeomorphism $h: M \rightarrow M$ (resp. $\Omega(f) \rightarrow \Omega(g)$ ) satisfying $g h=h f$.

Definition 1 [5]. We say that $f \in \operatorname{End}^{r}(M)$ satisfies Axiom A if there exist a continuous splitting $T M \mid \Omega(f)=E^{s} \oplus E^{u}$, and a Riemannian norm \|\| on $T M$, and constants $K>0,0<\lambda<1$ satisfying:

(a) $(T f) E^{s} \subseteq E^{s},(T f) E^{u}=E^{u}$;

(b) $\left\|(T f)^{n} v\right\| \leq K \lambda^{n}\|v\|$ for $x \in \Omega(f), v \in E_{x}^{s}, n>0$,

$$
\left\|(T f)^{n} v\right\| \geq K \lambda^{-n}\|v\| \quad \text { for } x \in \Omega(f), v \in E_{x}^{u}, n>0 ;
$$

(c) if $x_{1} \neq x_{2} \in \Omega(f)$ and $f\left(x_{1}\right)=f\left(x_{2}\right)=y$, then $E_{y}^{s}=\{0\}$;

(d) $\operatorname{Per}(f)$ is dense in $\Omega(f)$;

(e) $S(f) \cap \Omega(f)=\varnothing$.

Definition 2 [5]. Let $f \in \operatorname{End}^{r}(M)$ and let $\Lambda$ be a compact subset of $M$ with $f(\Lambda)=\Lambda$. We say that $\Lambda$ is a prehyperbolic set for $f$ if there exist a continuous splitting $T M \mid \Lambda=E^{s} \oplus E^{u}$, and a Riemannian norm \|\| on $T M$ and constants $K>0,0<\lambda<1$ satisfying:

(a) $(T f) E^{s} \subset E^{s},(T f) E^{u}=E^{u}$;

(b) $\left\|(T f)^{n} v\right\| \leq K \lambda^{n}\|v\|$ for $x \in \Lambda, v \in E_{x}^{s}, n>0$,

$$
\left\|(T f)^{n} v\right\| \geq K \lambda^{-n}\|v\| \quad \text { for } x \in \Lambda, v \in E_{x}^{u}, n>0 ;
$$

(c) if $x_{1} \neq x_{2} \in \Lambda$ and $f\left(x_{1}\right)=f\left(x_{2}\right)=y$, then $E_{y}^{s}=\{0\}$.

Moreover we need some definitions for infinitesimal stability. If $\Lambda$ is a compact subset of $M$ let $\Gamma^{b}(\Lambda)$ be the space of bounded sections of $T M \mid \Lambda$ with the norm $\|\eta\|=\sup \{\|\eta(x)\| \mid x \in \Lambda\}$ and let $\Gamma^{0}(\Lambda)$ be the closed subspace of continuous sections. If $f \in \operatorname{End}^{r}(M)$ and $f(\Lambda) \subset \Lambda$ let $T_{f} M \mid \Lambda$ be the pullback bundle of $T M \mid \Lambda$ by $f$. Let $\Gamma_{f}^{b}(\Lambda), \Gamma_{f}^{0}(\Lambda)$ be the corresponding spaces of bounded and continuous sections of $T_{f} M \mid \Lambda$. We define the linear operator $L_{f}: \Gamma^{b}(\Lambda) \rightarrow \Gamma_{f}^{b}(\Lambda)$ by

$$
L_{f}(\eta)=(T f) \circ \eta-\eta \circ f \quad \text { for } \eta \in \Gamma^{b}(\Lambda) .
$$

Definition 3. We say that $f \in \operatorname{End}^{r}(M)$ is infinitesimally stable if the linear operator $L_{f}: \Gamma^{0}(M) \rightarrow \Gamma_{f}^{0}(M)$ is surjective.

Remark. See [4] for definition of infinitesimal stability of diffeomorphisms. Infinitesimal stability of endomorphisms is a generalization of infinitesimal stability of diffeomorphisms. In fact, infinitesimally stable diffeomorphisms satisfy the definition of infinitesimal stability of endomorphisms. See Robbin $[14,15]$ and Mañé [6] for more detailed description of the case of diffeomorphisms.

It is easy to see that infinitesimal stability of diffeomorphisms is an open property. However, infinitesimal stability of endomorphisms is not an open property. Odani obtained the following:

Theorem B [11]. Let $f \in \operatorname{End}^{1}\left(S^{1}\right)$ be a homeomorphism such that $\Omega(f)=$ \{an attracting fixed point $a$ and a repelling fixed point $b\}$ and $S(f)=\{$ an attracting fixed point $a\}$. Then $f$ is infinitesimally stable.

Then we can get a noninfinitesimally stable endomorphism $g$ by an arbitrarily small $C^{1}$ perturbation around an attracting fixed point $a$ of $f$ in Theorem 
B. Hence we consider the interior of the set of infinitesimally stable endomorphisms. Let $\mathscr{F}^{r}(M)$ be the interior of the set of all $C^{r}$ infinitesimally stable endomorphisms of $M$. By the definition of infinitesimal stability of diffeomorphisms and the open mapping theorem of functional analysis, it is easy to see that $C^{r}$ infinitesimal stability of diffeomorphisms is an open property, $r \geq 1$. Hence it follows from [4] that Axiom A $C^{r}$ diffeomorphisms with the strong transversality condition belong to $\mathscr{F F}^{r}(M), r \geq 1$. Expanding maps also belong to $\mathscr{F}^{r}(M), r \geq 1$. For convenience we shall say that $f \in \operatorname{End}^{r}(M)$ is $S$-infinitesimally stable if $f$ belongs to $\mathscr{F F}^{r}(M)$.

\section{Proof of Theorem I}

In this section we will prove Theorem I. In the proof of the theorem, we shall use the following results.

Theorem C. Let $\Lambda$ be a prehyperbolic set for a $C^{r}$ endomorphism $f$ of $M$, $r \geq 1$. There are numbers $\alpha>0, K>0, k>0$, a neighborhood $U$ of $\Lambda$ in $M$ and a neighborhood $V$ of $f$ in $\operatorname{End}^{r}(M)$ with the following properties: For any topological space $X$, any homeomorphism $h$ of $X$, and any continuous map $i: X \rightarrow U$, if $g$ belongs to $V$ and $d(i h, g i)<\alpha$, then there is a unique continuous map $j: X \rightarrow M$ such that $j h=g j$ and $d(i, j) \leq k$. In fact, we have the stronger estimate that $d(i, j) \leq K d(i h, g i)$. Moreover, for fixed $i$ and $h, j$ depends $C^{0}$ continuously on $g$. Here $d(i, j)=\sup \{\rho(i(x), j(x)) \mid x \in$ $X\}$, where $\rho$ is a metric on $M$.

Theorem $\mathrm{C}$ is proved following the case of diffeomorphisms in Chapter 7 of [18, pp. 87-93].

Theorem D [3]. $C^{r}$ infinitesimally stable endomorphisms of $M$ satisfy weak Axiom $\mathrm{A}$ and the no cycles condition.

Here we say that $f \in \operatorname{End}^{r}(M)$ satisfies weak Axiom A if there exist a continuous splitting $T M \mid \Omega(f)=E^{s} \oplus E^{u}$, and a Riemannian norm \| $\|$ on $T M$, and constants $K>0,0<\lambda<1$ satisfying the conditions (a), (b), (c), (d) of the definition of Axiom A above. Note that $f$ in Theorem $\mathbf{B}$ is an example of infinitesimally stable endomorphisms which satisfy not Axiom A but weak Axiom A.

Let $\mathscr{F r}(M)$ be the interior of the set of all $C^{r}$ endomorphisms of $M$ such that every periodic point is hyperbolic. We say that a periodic point $x$ of $f \in \operatorname{End}^{r}(M)$ with period $p$ is prehyperbolic if $T f^{p}: T_{x} M \rightarrow T_{x} M$ has no eigenvalue of absolute value 1 . Let $\mathscr{P} \mathscr{F} r(M)$ be the interior of the set of all $C^{r}$ endomorphisms of $M$ such that every periodic point is prehyperbolic. It is obvious that $\mathscr{F}^{r}(M) \subset \mathscr{P} \mathscr{F}^{r}(M)$. By Theorem D, $\mathscr{F} \mathscr{F}^{r}(M) \subset \mathscr{P} \mathscr{F}^{r}(M)$. If $x$ is a prehyperbolic periodic point of $f$ with period $n$, let $E^{s}(x)$ and $E^{u}(x)$ be the stable and unstable subspaces of $T_{x} M$, i.e., the subspaces associated to the eigenvalues of $T f^{n}: T_{x} M \hookleftarrow$ that have respectively modulus $<1$ and $>1$. For $f \in \mathscr{P F} \mathscr{F}^{r}(M)$ let $P_{i}(f)$ be the set of points $x \in \operatorname{Per}(f)$ such that $\operatorname{dim} E^{s}(x)=i$, and denote by $N(i, n, f)$ the number of fixed points of $f^{n}$ contained in $P_{i}(f)$. We shall call the above number $i$ the stable index of periodic point $x$ for $f$. In the proof of Theorem I stable index will play an important role. 
The following lemma is a generalization of Lemma I.8 [7], and can be proved by the same argument for the case of diffeomorphisms in [7]. The main tools for the proof are fundamental results of a manifold of mappings [1], [17] and the implicit function theorem.

Lemma E. If $f \in \mathscr{P} \mathscr{F}^{r}(M)$ then there exists a $C^{r}$ neighborhood $\mathscr{U}$ of $f$ in $\operatorname{End}^{r}(M)$ such that:

(a) $N\left(i, n, g_{1}\right)=N\left(i, n, g_{2}\right)$ for all $g_{1} \in \mathscr{U}, g_{2} \in \mathscr{U}, n>0$ and $0 \leq i \leq$ $\operatorname{dim} M$.

(b) If $g \in \mathscr{U}$ and coincides with $f$ in a neighborhood of $\overline{P_{i}(f)}$ for some $0 \leq i \leq \operatorname{dim} M$, then $\overline{P_{i}(g)}=\overline{P_{i}(f)}$.

Remark. In fact, the above neighborhood $\mathscr{U}$ is contained in $\mathscr{P F}{ }^{r}(M)$.

Proof. Let $x$ be a periodic point of $f \in \mathscr{P} \mathscr{F}^{r}(M)$ with period $n$ and stable index $i$. Let $(\varphi, V)$ be a chart around $x$ such that $\varphi(x)=0$ and $\varphi(V) \subset R^{m}$ where $m=\operatorname{dim} M$. Then we define $F: \operatorname{End}^{r}(M) \times V \rightarrow M$ by $F(g, y)=$ $g^{n}(y)$. It is easy to see that $F$ is $C^{r}[1,17]$. Let $U_{1}(f) \subset \operatorname{End}^{r}(M)$ be an open neighborhood of $f$ such that $g^{n}\left(V^{\prime}\right) \subset V$ for all $g \in U_{1}(f)$ where $V^{\prime}$ is an open neighborhood of $x$ with $V^{\prime} \subset V$. Then $F: U_{1}(f) \times V^{\prime} \rightarrow V$ is well defined and $C^{r}$. Let $U_{1}^{*}(f) \subset \Gamma^{r}\left(f^{*} T M\right)$ be an open neighborhood of zero section of $f^{*} T M$ such that $U_{1}^{*}(f)$ can be identified with $U_{1}(f)$ by a diffeomorphism $G$ defined by $G(\xi)(x)=\exp _{f(x)} \xi(x)$. Then $\widehat{F}: U_{1}^{*}(f) \times$ $\varphi\left(V^{\prime}\right) \rightarrow R^{m}$ is defined by

$$
\widehat{F}(\xi, y)=\varphi \circ(G(\xi))^{n} \circ \varphi^{-1}(y)-y .
$$

It is obvious that $\widehat{F}$ is $C^{r}$ and $\widehat{F}(0,0)=0$. Moreover, $D_{2} \widehat{F}(0,0)$ is an isomorphism of $R^{m}$ because $f \in \mathscr{P} \mathscr{F}^{r}(M)$. By the implicit function theorem, there exist open neighborhoods $U_{1}^{*} \subset U_{1}^{*}(f)$ of zero section in $\Gamma^{r}\left(f^{*} T M\right)$, $V^{*} \subset \varphi\left(V^{\prime}\right)$ of 0 in $R^{m}$ such that, for very open connected neighborhood $U_{2}^{*}$ of zero section, contained in $U_{1}^{*}$, there is a unique continuous map $h$ of $U_{2}^{*}$ into $V^{*}$ such that $h(0)=0, \widehat{F}(\eta, h(\eta))=0$ for any $\eta \in U_{2}^{*}$. Then we can take $U_{1}^{*}$ as an open connected neighborhood of zero section. Moreover, we can take $U_{1}^{*}$ with the following property: let $U=\left\{g \in \operatorname{End}^{r}(M) \mid g=G(\eta)\right.$ for $\left.\eta \in U_{1}^{*}\right\}, \mathrm{Cl}(U) \subset \mathscr{P} \mathscr{F}^{r}(M)$. Then

$$
\widehat{F}(\eta, h(\eta))=\varphi \circ g_{\eta}^{n}\left(\varphi^{-1}(h(\eta))\right)-h(\eta)=0
$$

for every $\eta \in U_{1}^{*}$, where $g_{\eta}=G(\eta)$. Therefore $\varphi \circ g_{\eta}^{n}\left(\varphi^{-1}(h(\eta))\right)=h(\eta) \in$ $V^{*} \subset \varphi\left(V^{\prime}\right)$, so $g_{\eta}^{n}\left(\varphi^{-1}(h(\eta))\right)=\varphi^{-1}(h(\eta)) \in V^{\prime}$. That is, $\varphi^{-1}(h(\eta))$ is a periodic point of $g_{\eta}=G(\eta)$ with period $n$ and stable index $i$ for every $\eta \in U_{1}^{*}$ (because $\left.g_{\eta} \in \mathscr{P} \mathscr{F}^{r}(M)\right)$. Now take a continuous arc $g(t)$ such that $g(0)=f$, $g(t) \in U$ for all $0 \leq t<1$, and $g(1) \in \partial U$. Of course, $g(t) \in \bar{U} \subset \mathscr{P} \mathscr{F}^{r}(M)$ for all $0 \leq t \leq 1$. Hence $g(1)$ has a prehyperbolic periodic point $z$ with period $n$ and stable index $i$ satisfying $z=\lim _{t \rightarrow 1} \varphi^{-1}\left(h\left(G^{-1}(g(t))\right)\right)$. We repeat the above argument for $g(1)$ and $z$ instead of $f$ and $x$. Hence we can get that $N(i, n, f) \leq N(i, n, g)$ if there exists a continuous arc $g(t)$ such that $g(t) \in \mathscr{P} \mathscr{F}^{r}(M)$ for all $0 \leq t \leq 1$ and $g(0)=f, g(1)=g$. Reversing the 
roles of $f$ and $g$, it follows that $N(i, n, g) \leq N(i, n, f)$. Therefore if $f$ and $g$ are in a connected component of $\mathscr{P} \mathscr{F}^{r}(M)$ then $N(i, n, f)=N(i, n, g)$ for all $n \geq 1$ and $0 \leq i \leq \operatorname{dim} M$. See [7] for the proof of (b).

Proof of Theorem I. Since $f \in \mathscr{F F}^{r}(M)$, there exists a $C^{r}$ neighborhood $\mathscr{U}$ of $f$ in $\operatorname{End}^{r}(M)$ such that every $g \in \mathscr{U}$ is infinitesimally stable. By Theorem $\mathrm{D}$, it is easy to see that $\mathscr{U} \subset \mathscr{P} \mathscr{F}^{r}(M)$. Moreover, for each $g \in \mathscr{U} \Omega(g)$ has a decomposition, $\Lambda_{0}(g) \cup \Lambda_{1}(g) \cup \cdots \cup \Lambda_{\operatorname{dim} M}(g)$ into disjoint prehyperbolic sets. Here each $\Lambda_{i}(g)$ is a prehyperbolic set such that it coincides with the closure of the prehyperbolic periodic points of $g$ with stable index $i$. We shall show that there exists a $C^{r}$ neighborhood $\mathscr{U}_{1} \subset \mathscr{U}$ of $f$ such that for every $g \in \mathscr{U}_{1}$, $f \mid \Lambda_{i}(f)$ is topologically conjugate to $g \mid \Lambda_{i}(g)$ for all $0 \leq i \leq \operatorname{dim} M$. Since $f \mid \Lambda_{0}(f)$ is a (quasi-)expanding map, there exist neighborhoods $U_{0}$ of $\Lambda_{0}(f)$ and $\mathscr{U}^{0}$ of $f$ such that if $g \in \mathscr{U}^{0}$ then there exists a homeomorphism $h$ from $\Lambda_{0}(f)$ onto its image satisfying $g h=h f$ and $h\left(\Lambda_{0}(f)\right) \subset U_{0}$ [13]. Then $h\left(\Lambda_{0}(f)\right)=\Lambda_{0}(g)$ because $f \in \mathscr{P}^{r}(M)$ and Lemma E. For $1 \leq i \leq \operatorname{dim} M$ we apply Theorem $C$ to each $\Lambda_{i}(f)$. Then there exist neighborhoods $U_{i}$ of $\Lambda_{i}(f)$ and $V_{i}$ of $f$ and constants $\alpha_{i}, k_{i}$, and $K_{i}$ as in Theorem C. If $g$ satisfies $d_{C^{0}}(f, g)<\alpha_{i}, d\left(g \circ\right.$ inc $_{i}$, inc $\left._{i} \circ f\right)<\alpha_{i}$ where inc $_{i}: \Lambda_{i}(f) \rightarrow$ $M$ is the inclusion map, $d_{C^{0}}(f, g)=\sup \{\rho(f(x), g(x)) \mid x \in M\}, d$ is a metric on $C^{0}\left(\Lambda_{i}(f), M\right)$, that is, $d(a, b)=\sup \left\{\rho(a(x), b(x)) \mid x \in \Lambda_{i}(f)\right\}$. Hence if $g \in V_{i}, d_{C^{0}}(f, g)<\alpha_{i}$ then there exists a unique continuous map $\Phi_{i}(g): \Lambda_{i}(f) \rightarrow M$ satisfying $d\left(\right.$ inc $\left._{i}, \Phi_{i}(g)\right) \leq k_{i}$, and $g \Phi_{i}(g)=\Phi_{i}(g) f$. Furthermore, we know that $\Phi_{i}(g)$ depends continuously on $g$ and satisfies $d\left(\right.$ inc $\left._{i}, \Phi_{i}(g)\right) \leq K_{i} d_{C^{0}}(f, g)$. It is easy to see that $\Phi_{i}(f)=i n c_{i}$. Let $W_{i} \subset$ $V_{i} \cap \mathscr{U}$ be a $C^{r}$ connected neighborhood of $f$ such that for every $g \in W_{i}$ the set $\Phi_{i}(g)\left(\Lambda_{i}(f)\right)$ is contained in $U_{i}$. Observe that $\Phi_{i}(g)\left(P_{i}(f)\right)$ is a set of periodic points of $g$, and $\Phi_{i}(g)\left(\Lambda_{i}(f)\right)=\overline{\Phi_{i}(g)\left(P_{i}(f)\right)}$ for $g \in W_{i}$. We shall show that $\Phi_{i}\left(\Lambda_{i}(f)\right) \subset \Lambda_{i}(g)=\mathrm{Cl}\left(P_{i}(g)\right)$ for every $g \in W_{i}$. Let $g(t) \in W_{i}$, $0 \leq t \leq 1$, be a continuous arc with $g(0)=f$ and $g(1)=g$. Then if $x$ is a periodic point of $f$ with period $p$ then $\Phi_{i}(g(t))(x)$ is a periodic point of $g(t)$ with period $p$. Since $g(t) \in \mathscr{P} \mathscr{F}^{r}(M)$ for all $0 \leq t \leq 1$, the stable index of $x$ for $f$ is equal to that of $\Phi_{i}(g(t))(x)$ for $g(t)$. Hence $\Phi_{i}(g)\left(P_{i}(f)\right) \subset$ $P_{i}(g)$ so $\Phi_{i}(g)\left(\Lambda_{i}(f)\right) \subset \Lambda_{i}(g)$. Let $\Lambda_{g}=\Phi_{i}(g)\left(\Lambda_{i}(f)\right)$. Then $\Lambda_{g}$ is a $g$-invariant compact subset of $\Lambda_{i}(g)$. Since $\Lambda_{i}(g)$ is a prehyperbolic set for $g$ and $i>0, g \mid \Lambda_{g}$ is a homeomorphism from $\Lambda_{g}$ to itself. By Theorem $\mathrm{C}$, there exists a unique continuous map $j: \Lambda_{g} \rightarrow M$ such that $j g=f j$ and $d\left(\mathrm{id} \mid \Lambda_{g}, j\right) \leq K_{i} d_{C^{0}}(f, g)$. We also see that $d\left(i n c_{i}, j \Phi_{i}(g)\right) \leq 2 K_{i} d_{C^{0}}(f, g)$, $\left[j \circ \Phi_{i}(g)\right] \circ f=f \circ\left[j \circ \Phi_{i}(g)\right]$. Let $\mathscr{U}^{i} \subset W_{i}$ be a $C^{r}$ neighborhood of $f$ such that if $g \in \mathscr{U}^{i}$ then $2 K_{i} d_{C^{0}}(f, g) \leq k_{i}$. If $g \in \mathscr{U}^{i}$, then we have $j \circ \Phi_{i}(g)=\mathrm{id} \mid \Lambda_{i}(f)$ so $\Phi_{i}(g)$ is injective. Hence $\Phi_{i}(g): \Lambda_{i}(f) \rightarrow \Lambda_{g}$ is a homeomorphism. Therefore $\Phi_{i}(g) P_{i}(f)=P_{i}(g)$ because Lemma E. Hence $\Lambda_{g}=\mathrm{Cl}\left(\Phi_{i}(g) P_{i}(f)\right)=\Lambda_{i}(g)$. If necessary, we take smaller neighborhoods $\mathscr{U}^{i}, 0 \leq i \leq \operatorname{dim} M$, so that we can take a disjoint family $\left\{U_{i} \mid 0 \leq i \leq \operatorname{dim} M\right\}$. Let $\mathscr{U}_{1}=\bigcap_{i=0}^{\operatorname{dim} M} \mathscr{U}^{i}$. Then for every $g \in \mathscr{U}_{1}, f \mid \Omega(f)$ is topologically conjugate to $g \mid \Omega(g)$.

By analogous argument to the proof of Theorem I, we obtain the following result: 
Lemma F. Let $\operatorname{Diff}^{r}(M)$ be the space of $C^{r}$ diffeomorphisms of $M$ endowed with the $C^{r}$ topology, $r \geq 1$. Let $f \in \operatorname{Diff}^{r}(M)$. Then the following are equivalent:

(a) $f$ satisfies Axiom A and the no cycles condition;

(b) $f$ has a $C^{r}$ neighborhood $\mathscr{N}$ in $\operatorname{Diff}^{r}(M)$ such that every $g$ in $\mathscr{N}$ satisfies Axiom A.

Our sufficient condition for $\Omega$-stability in Corollary I corresponds to (b) in Lemma F. Our sufficient condition is weaker than Przytycki's conditions. Because our condition plus $\Omega(f) \cap S(f)=\varnothing$ is equivalent to Axiom A plus the nocycles condition (that is, Przytycki's conditions). Hence Corollary I is a natural generalization of Smale's $\Omega$-stability theorem [19].

Remark. It is not known whether our sufficient condition is equivalent to weak Axiom A plus the no cycles condition.

\section{Proof of TheOREM II}

In this section we shall prove Theorem II. We begin with a definition following Przytycki [12].

Definition. We say that $f \in \operatorname{End}^{r}(M)$ is an Anosov endomorphism if $S(f)=\varnothing$ and there exist constants $K>0,0<\lambda<1$, and a Riemannian norm \|\| on $T M$ such that for every $f$-orbit $\left(x_{n}\right)$ there exists a splitting of $\bigcup_{n=-\infty}^{\infty} T_{x_{n}} M=$ $E^{s} \oplus E^{u}=\bigcup_{n=-\infty}^{\infty} E_{x_{n}}^{s} \oplus E_{x_{n}}^{u}$ satisfying:

(a) $(T f) E^{s}=E^{s},(T f) E^{u}=E^{u}$;

(b) $\left\|(T f)^{n} v\right\| \leq K \lambda^{n}\|v\|$ for $v \in E^{s}, n \geq 0$,

$$
\left\|(T f)^{n} v\right\| \geq K^{-1} \lambda^{-n}\|v\| \quad \text { for } v \in E^{u}, n \geq 0 .
$$

In the proof of Theorem II we use the following lemma.

Lemma G [5]. Let $f \in \operatorname{End}^{r}(M)$ and let $\Lambda$ be a compact subset of $M$ with $f(\Lambda)=\Lambda$. Then $\Lambda$ is a prehyperbolic set for $f$ if and only if $L_{f}: \Gamma^{0}(\Lambda) \rightarrow$ $\Gamma_{f}^{0}(\Lambda)$ is an isomorphism.

Proof. "If part" is proved by the same arguments in the proof of Lemma A [5]. We only need to prove "only if part". Suppose that $\Lambda$ is a prehyperbolic set for $f$. Without loss of generality we can suppose that $\Lambda$ cannot be decomposed into disjoint prehyperbolic sets. At first we shall show the injectivity of $L_{f}$. Suppose that $L_{f}: \Gamma^{0}(\Lambda) \rightarrow \Gamma_{f}^{0}(\Lambda)$ is not injective. Then there exists $0 \neq$ $\eta \in \Gamma^{0}(\Lambda)$ with $L_{f}(\eta)=0$. Hence there exists an $f$-orbit $\left(x_{n}\right)$ such that $\eta\left(x_{0}\right) \neq 0$. Then $\left\{\eta\left(x_{n}\right)\right\} \subset T M$ is a bounded sequence such that $\eta\left(x_{n}\right) \in$ $T_{x_{n}} M$ and $(T f) \eta\left(x_{n}\right)=\eta\left(x_{n+1}\right)$ for all $n \in Z$. Since $\Lambda$ is prehyperbolic, $\eta\left(x_{0}\right) \in E_{x_{0}}^{s}$. Moreover $\left\|\eta\left(x_{-n}\right)\right\| \geq K^{-1} \lambda^{-n}\left\|(T f)^{n} \eta\left(x_{-n}\right)\right\|=K^{-1} \lambda^{-n}\left\|\eta\left(x_{0}\right)\right\|$ for all $n>0$. Hence $\left\{\left\|\eta\left(x_{-n}\right)\right\| \mid n>0\right\}$ is unbounded. This is a contradiction. Therefore $L_{f}$ is injective. Next we shall show that $L_{f}$ is surjective. Since $\Lambda$ is prehyperbolic for $f$, we can decompose $\Gamma^{0}(\Lambda)=\Gamma^{0}(\Lambda)^{s} \oplus \Gamma^{0}(\Lambda)^{u}$ and $\Gamma_{f}^{0}(\lambda)=\Gamma_{f}^{0}(\Lambda)^{s} \oplus \Gamma_{f}^{0}(\Lambda)^{u}$ where if $\eta \in \Gamma^{0}(\Lambda)^{s}$ then $\eta(x) \in E_{x}^{s}$ for all $x \in \Lambda$, similarly for $\Gamma^{0}(\Lambda)^{u}, \Gamma_{f}^{0}(\Lambda)^{s}, \Gamma_{f}^{0}(\Lambda)^{u}$. It is obvious that $L_{f}\left(\Gamma^{0}(\Lambda)^{s}\right) \subset \Gamma_{f}^{0}(\Lambda)^{s}$, $L_{f}\left(\Gamma^{0}(\Lambda)^{u}\right) \subset \Gamma_{f}^{0}(\Lambda)^{u}$. We shall show that $L_{f}: \Gamma^{0}(\Lambda)^{u} \rightarrow \Gamma_{f}^{0}(\Lambda)^{u}$ is surjective. 
Let $\xi \in L_{f}^{0}(\Lambda)^{u}$. We define a bounded section $\eta^{u} \in \Gamma^{b}(\Lambda)^{u}$ by

$$
\eta^{u}(x)=\sum_{k=1}^{\infty}\left(T f^{k} \mid E_{x}^{u}\right)^{-1} \xi\left(f^{k-1}(x)\right)
$$

Since $\Lambda$ is prehyperbolic for $f,\left\|(T f)^{n} v\right\| \geq K \lambda^{-n}\|v\|$ for all $v \in E^{u}$ and all $n>0$. Hence $\left\|\left(T f^{n} \mid E_{x}\right)^{-1}\right\| \leq K^{-1} \lambda^{n}$ for all $x \in \Lambda$ and $n>0$. Then $\left\|\eta^{u}(x)\right\|=\sum_{k=1}^{\infty}\left\|\left(T f^{k} \mid E_{x}^{u}\right)^{-1}\right\|\|\xi\| \leq \sum_{k=1}^{\infty} K^{-1} \lambda^{k}\|\xi\|=\frac{K^{-1}\|\xi\|}{1-\lambda}$. Therefore $\eta^{u}$ is bounded. Moreover it is easy to see that $L_{f}\left(\eta^{u}\right)=\xi$. We prove the continuity of $\eta^{u}$. Let $\varepsilon>0$ be arbitrarily small. Let us take $\delta>0$ and $1>\mu>0$ such that if $d(x, y)<\delta$ then

(i) $\left\|\pi_{x_{i+1}}^{u} \circ \varepsilon_{x_{i+1}, y_{i+1}} \circ \xi\left(y_{i}\right)-\xi\left(x_{i}\right)\right\|<\mu$,

(ii) $\left\|\varepsilon_{x_{i}, y_{i}} \circ\left(T f \mid E_{y_{i}}^{u}\right)^{-1}-\left(T f \mid E_{x_{i}}^{u}\right)^{-1} \circ \pi_{x_{i+1}}^{u} \circ \varepsilon_{x_{i+1}, y_{i+1}}\right\|<\mu$,

(iii) $\max \left\{\left\|\varepsilon_{y_{i}, x_{i}}\right\|,\left\|\varepsilon_{x_{i}, y_{i}}\right\| \mid 0 \leq i \leq n\right\}<\frac{3}{2}$,

for all $0 \leq i \leq n-1$, where $n$ is a positive integer, $\varepsilon_{x y}$ is the parallel translation from $T_{y} M$ into $T_{x} M$ along the unique shortest geodesic joining $x$ to $y, \pi_{x}^{u}$ is a projection from $T_{x} M$ to $E_{x}^{u}$ along $E_{x}^{s}, d($,$) is the metric on M$ associated to the Riemannian metric.

Then, for $y$ satisfying $d(x, y)<\delta$,

$$
\begin{aligned}
& \left\|\varepsilon_{x y} \eta^{u}(y)-\eta^{u}(x)\right\| \\
& =\left\|\varepsilon_{x y}\left\{\left(T f^{n} \mid E_{y}^{u}\right)^{-1} \eta^{u}\left(y_{n}\right)+\sum_{k=1}^{n}\left(T f^{k} \mid E_{y}^{u}\right)^{-1} \xi\left(y_{k-1}\right)\right\}-\sum_{k=1}^{\infty}\left(T f^{k}\right)^{-1} \xi\left(x_{k-1}\right)\right\| \\
& \leq \sum_{k=1}^{n}\left\|\varepsilon_{x y} \circ\left(T f^{k} \mid E_{y}^{u}\right)^{-1} \circ \xi\left(y_{k-1}\right)-\left(T f^{k} \mid E_{x}^{u}\right)^{-1} \circ \xi\left(x_{k-1}\right)\right\| \\
& \quad+\sum_{k=n+1}^{\infty}\left\|\left(T f^{k} \mid E_{x}^{u}\right)^{-1} \circ \xi\left(x_{k-1}\right)\right\|+\left\|\varepsilon_{x y} \circ\left(T f^{n} \mid E_{y}^{u}\right)^{-1} \circ \eta^{u}\left(y_{n}\right)\right\| .
\end{aligned}
$$

On the other hand,

$$
\begin{aligned}
& \left\|\varepsilon_{x y} \circ\left(T f^{k} \mid E_{y}^{u}\right)^{-1} \circ \xi\left(y_{k-1}\right)-\left(T f^{k} \mid E_{x}^{u}\right)^{-1} \circ \xi\left(x_{k-1}\right)\right\| \\
& \leq\left\|\varepsilon_{x y} \circ\left(T f^{k} \mid E_{y}^{u}\right)^{-1} \circ \xi\left(y_{k-1}\right)-\left(T f^{k} \mid E_{x}^{u}\right)^{-1} \circ \pi_{x_{k}}^{u} \circ \varepsilon_{x_{k} y_{k}} \xi\left(y_{k-1}\right)\right\| \\
& \quad+\left\|\left(T f^{k} \mid E_{x}^{u}\right)^{-1} \circ \pi_{x_{k}}^{u} \varepsilon_{x_{k} y_{k}} \xi\left(y_{k-1}\right)-\left(T f^{k} \mid E_{x}^{u}\right)^{-1} \circ \xi\left(x_{k-1}\right)\right\| \\
& \leq\left\|\varepsilon_{x y} \circ\left(T f^{k} \mid E_{y}^{u}\right)^{-1}-\left(T f^{k} \mid E_{x}^{u}\right)^{-1} \circ \pi_{x_{k}}^{u} \circ \varepsilon_{x_{k} y_{k}}\right\|\left\|\xi\left(y_{k-1}\right)\right\| \\
& \quad+\left\|\left(T f^{k} \mid E_{x}^{u}\right)^{-1}\right\|\left\|\pi_{x_{k}}^{u} \circ \varepsilon_{x_{k} y_{k}} \xi\left(y_{k-1}\right)-\xi\left(x_{k-1}\right)\right\| \\
& \leq\left\|\xi\left(y_{k-1}\right)\right\| \sum_{i=1}^{k}\left\|\varepsilon_{x y} \circ\left(T f^{i-1} \mid E_{y}^{u}\right)^{-1}\right\| \|\left(T f \mid E_{y_{i-1}}^{u}\right)^{-1} \circ \pi_{y_{i}}^{u} \circ \varepsilon_{y_{i} x_{i}}-\varepsilon_{y_{i-1} x_{i-1}} \\
& \quad \circ\left(T f \mid E_{x_{i}}^{u}\right)^{-1}\|\|\left(T f^{k-i} \mid E_{x_{i}}^{u}\right)^{-1}\|\| \varepsilon_{x_{k} y_{k}} \|+K^{-1} \lambda^{k} \mu \\
& \leq\left(\frac{3}{2}\right)^{2}\|\xi\| \sum_{i=1}^{k}\left(K^{-1} \lambda^{i-1}\right) \mu\left(K^{-1} \lambda^{k-i}\right)+K^{-1} \lambda^{k} \mu
\end{aligned}
$$




$$
\begin{aligned}
& =\left(\frac{3}{2}\right)^{2}\|\xi\| K^{-2} \mu \sum_{i=1}^{k} \lambda^{k-1}+K^{-1} \lambda^{k} \mu \\
& =\left(\frac{3}{2}\right)^{2}\|\xi\| K^{-2} \mu k \lambda^{k-1}+K^{-1} \lambda^{k} \mu .
\end{aligned}
$$

Hence,

$$
\begin{aligned}
& \left\|\varepsilon_{x y} \eta^{u}(y)-\eta^{u}(x)\right\| \leq\left(\frac{3}{2}\right)^{2}\|\xi\| K^{-2} \mu(1-\lambda)^{-2}+K^{-1} \mu \lambda(1-\lambda)^{-1} \\
& \quad+K^{-1}\|\xi\| \sum_{k=n+1}^{\infty} \lambda^{k}+\frac{3}{2} K^{-2} \lambda^{n}\|\xi\| \\
& \leq\left(\frac{3}{2}\right)^{2}\|\xi\| K^{-2} \mu(1-\lambda)^{-2}+K^{-1} \mu \lambda(1-\lambda)^{-1} \\
& \quad+K^{-1}\|\xi\| \lambda^{n+1}(1-\lambda)^{-1}+\frac{3}{2} K^{-2} \lambda^{n}(1-\lambda)^{-1}\|\xi\| .
\end{aligned}
$$

Therefore given $\xi \in \Gamma_{f}^{0}(\Lambda)^{u}$ and $\varepsilon>0$ we take $1>\mu>0$ and $N \in Z^{+}$such that

(a) $\left(\frac{3}{2}\right)^{2}\|\xi\| K^{-2} \mu(1-\lambda)^{-2}<\frac{\varepsilon}{4}$,

(b) $K^{-1} \mu \lambda(1-\lambda)^{-1}<\frac{\varepsilon}{4}$,

(c) $K^{-1}\|\xi\| \lambda^{N+1}(1-\lambda)^{-1}<\frac{\varepsilon}{4}$,

(d) $\frac{3}{2} K^{-2} \lambda^{N}\|\xi\|<\frac{\varepsilon}{4}$.

Let $n$ be a positive integer such that $n \geq N$. Then we can take $\delta>0$ satisfing (i), (ii), (iii) for $\mu, n$ above. So if $d(x, y)<\delta$ then $\left\|\eta^{u}(x)-\varepsilon_{x y} \eta^{u}(y)\right\|<\varepsilon$. Hence $\eta^{u}$ is uniformly continuous. So if $E^{s}=0$ then $L_{f}: \Gamma^{0}(\Lambda) \rightarrow \Gamma_{f}^{0}(\Lambda)$ is surjective. If $E^{s} \neq 0$ then $f \mid \Lambda$ is bijective. Then for each $x \in \Lambda$ there exists a unique $f$-orbit $\left(x_{n}\right)$ such that $x_{0}=x, f\left(x_{n}\right)=x_{n+1}$ and $x_{n} \in \Lambda$ for all $n \in Z$.

Let $\xi^{s} \in \Gamma_{f}^{0}(\Lambda)^{s}$. We define a bounded section $\eta^{s} \in \Gamma^{b}(\Lambda)^{s}$ by

$$
\eta^{s}(x)=-\sum_{k=0}^{\infty} T f^{k}\left(x_{-k}\right) \xi^{s}\left(x_{-k-1}\right) .
$$

Then $L_{f}\left(\eta^{s}\right)=\xi^{s}$. The continuity of $\eta^{s}$ is proved by the similar estimate to that for the continuity of $\eta^{u}$ above. Hence $L_{f}: \Gamma^{0}(\Lambda)^{s} \rightarrow \Gamma_{f}^{0}(\Lambda)^{s}$ is surjective.

Proof of Theorem II. (b) $\Rightarrow$ (a). By [9] an Anosov diffeomorphism is structurally stable. And an expanding map is structurally stable [17].

"(a) $\Rightarrow(\mathrm{b})$ " is proved in [12].

(b) $\Rightarrow$ (c). If $f$ is either an Anosov diffeomorphism or an expanding map, then $M$ is a prehyperbolic set for $f$. By Lemma $G, L_{f}: \Gamma^{0}(M) \rightarrow \Gamma_{f}^{0}(M)$ is surjective, that is, $f$ is infinitesimally stable. Moreover there exists a neighborhood $\mathscr{U}$ of $f$ such that every $g \in \mathscr{U}$ is either an Anosov diffeomorphism or an expanding map. Hence $f$ belongs to $\mathscr{F F}^{r}(M)$.

(c) $\Rightarrow(b)$. Suppose that an Anosov endomorphism $f$ is not an expanding map. Then $f$ is a proper Anosov endomorphism. Hence there exist constants 
$K>0,0<\lambda<1$, and a Riemannian norm \|\| on $T M$ such that for every $f$ orbit $\left(x_{n}\right)$ there exists a splitting of $\bigcup_{n=-\infty}^{\infty} T_{x_{n}} M=E^{s} \oplus E^{u}=\bigcup_{n=-\infty}^{\infty} E_{x_{n}}^{s} \oplus E_{x_{n}}^{u}$ satisfying

(i) $(T f) E^{s}=E^{s},(T f) E^{u}=E^{u}$,

(ii) $\left\|(T f)^{n} v\right\| \leq K \lambda^{n}\|v\|$ for $v \in E^{s}, n \geq 0$,

$$
\left\|(T f)^{n} v\right\| \geq K^{-1} \lambda^{-n}\|v\| \quad \text { for } v \in E^{u}, n \geq 0,
$$

(iii) $\operatorname{dim} E_{x}^{s}=$ constant $>0$ for all $x \in\left(x_{n}\right)$ and all $f$-orbits $\left(x_{n}\right)$.

If $f$ is injective, then $f$ is an Anosov diffeomorphism. So we suppose that $f$ is not injective. Using (i) and (ii), it is easy to see that $L_{f}$ is injective. Hence $L_{f}: \Gamma^{0}(M) \rightarrow \Gamma_{f}^{0}(M)$ is an isomorphism. By Lemma G, $M$ is a prehyperbolic set for $f$. Since $f$ is not injective, $f$ is an expanding map contradicting to (iii).

Corollary II. Let $f$ be a $C^{r}$ Anosov endomorphism of $M$. Then $f$ is $S$ infinitesimally stable if and only if it is infinitesimally stable.

\section{CONCLUding REMARKS}

Comparing Theorem A with Theorem II, we induce the following conjecture.

Conjecture I. Let $f$ be a $C^{1}$ endomorphism of $M$ where $M$ is a closed smooth manifold with $\operatorname{dim} M \geq 2$. Then the following are equivalent:

(a) $f$ is $C^{1}$ structurally stable;

(b) $f$ is either an AS-diffeomorphism or an expanding map;

(c) $f$ is S-infinitesimally stable.

Here we call $f$ an $A S$-diffeomorphism if $f$ satisfies Axiom A and the strong transversality condition $[4,14]$. In [8] the following conjecture was stated:

Conjecture II. If $f \in \operatorname{End}^{r}(M)$ is $C^{r}$ structurally stable, then $\Omega(f) \cap S(f)=\varnothing$.

Relevant partial results were obtained by $[5,2]$. In order to investigate the relation between structural stability and $S$-infinitesimal stability, we may pose the following problem analogous to Conjecture II.

Problem. If $f$ belongs to $\mathscr{F F}^{r}(M)$, then $\Omega(f) \cap S(f)=\varnothing$.

From this point of view, the following relevant results were obtained.

Proposition A [11]. $C^{r}$ infinitesimally stable endomorphisms of $S^{1}$ are strictly monotone, $r \geq 1$.

Proposition B [10]. $C^{\infty}$ infinitesimally stable endomorphisms of $S^{1}$ have no fold points.

Proposition C. $C^{\infty}$ infinitesimally stable endomorphisms of $M$ with $\operatorname{dim} M=$ 2 have no fold points and no cusps.

It follows from Theorem $\mathrm{D}$ and Proposition A that $C^{1} S$-infinitesimally stable endomorphisms of $S^{1}$ satisfy Axiom A and have no singularities. It is well known that $C^{1}$ structurally stable endomorphisms of $S^{1}$ satisfy Axiom A and have no singularities. 


\section{REFERENCES}

1. R. Abraham and J. Robbin, Transversal mappings and flows, Benjamin, New York, 1967.

2. H. Ikeda, On stability of endomorphisms, Proc. Amer. Math. Soc. 104 (1988), 1287-1290.

3. _ On infinitesimal stability of endomorphisms, The Study of Dynamical Systems, vol. 4, World Scientific, Singapore, 1989, pp. 59-83.

4. R. Mañé, On infinitesimal and absolute stability of diffeomorphisms, Dynamical Systems (Warwick, 1974), Lecture Notes in Math., vol. 468, Springer-Verlag, Berlin, Heidelberg and New York, 1975, pp. 151-161.

5. __ Axiom A for endomorphisms, Lecture Notes in Math., vol. 597, Springer-Verlag, Berlin, Heidelberg and New York, 1977, pp. 379-388.

6. _ Characterizations of AS diffeomorphisms, Lecture Notes in Math., vol. 597, Springer-Verlag, Berlin, Heidelberg and New York, 1977, pp. 389-394.

7. $\ldots$ A proof of the $C^{1}$ stability conjecture, Publ. Math. Inst. Hautes Etude Sci. 66 (1987), 161-210.

8. R. Mañé and C. Pugh, Stability of endomorphisms, Lecture Notes in Math., vol. 468, Springer-Verlag, Berlin, Heidelberg and New York, 1975, pp. 175-184.

9. J. Moser, On a theorem of Anosov, J. Differential Equations 5 (1969), 411-440.

10. I. Nakai, A personal communication.

11. K. Odani, A personal communication.

12. F. Przytycki, Anosov endomorphisms, Studia Math. 58 (1976), 249-285.

13. $\_$, On $\Omega$-stability and structural stability of endomorphisms satisfying Axiom A, Studia Math. 60 (1977), 61-77.

14. J. Robbin, A structural stability theorem, Ann. of Math. (2) 94 (1971), 447-493.

15. __ Topological conjugacy and structural stability for discrete dynamical systems, Bull. Amer. Math. Soc. 78 (1972), 923-952.

16. C. Robinson, Structural stability of $C^{1}$ diffeomorphisms, J. Differential Equations 22 (1976), 28-73.

17. M. Shub, Endomorphisms of compact differentiable manifolds, Amer. J. Math. 91 (1969), 175-199.

18. _ Global stability of dynamical systems, Springer-Verlag, Berlin and New York, 1987.

19. S. Smale, The $\Omega$-stability theorem, Proc. Sympos. Pure Math., vol. 14, Amer. Math. Soc., Providence, RI, 1970, pp. 289-297.

Department of Mathematics, School of Education, Waseda University, Shinjuku, TOKYO, 169-50, JAPAN 